\title{
Analysis of Radio diagnostic test referrals in government hospitals
}

\author{
Surinder Pal ${ }^{1}$, Amarjeet Singh ${ }^{2}$, Akshay Anand ${ }^{3 *}$ \\ ${ }^{1}$ Centre for Systems Biology and Bioinformatics, Punjab University, Chandigarh, India

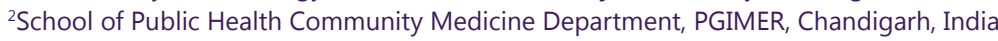 \\ ${ }^{3}$ Neuroscience Research Lab, Department of Neurology, PGIMER, Chandigarh, India
}

\section{KEY WORDS}

\section{Radiology}

Diagnostics test

Healthcare System

Outsourcing

*Corresponding Author:

Akshay Anand, PhD

Neuroscience Research Lab

Department of Neurology, PGIMER, Chandigarh, India

Contact no: +91-9815968102

E-mail: akshay1anand@rediffmail.com

In any treatment taken by patient, cost is an important dimension. These days, diagnostic tests comprise of a significant part of the health cost borne by patient for the treatment. Catastrophic expenditure is a commonly discussed issue by the health care administration. It is often argued that, outsourcing of the medical diagnostic tests is quite frequent. Daniel et al observed in their research survey that there were so many institutes which were outsourcing the tests. They concluded that it may be due to lack of resources in radiology lab [1]. Therefore, in order to avoid the rush of patients or waiting for a long period of time, patient himself or herself prefers to go outside the medical hospital for the tests. The public discourse is rather focused on how to overcome the capacity deficits in the public health system, rather than consolidating the existing resources and data from huge patient rush. This restricts access to vital health information which is being rapidly lost due to outsourcing of diagnostic tests referred by the hospitals, either due to lobbying by private labs or lack of policy in the matter, or both.

A study showed that private labs carried out clinical mistakes in the tests, showing that there is no one accountable and this may lead to out of pocket expenses of patients [2]. Friedman [3] and Feinberg et al [4] have found in a survey carried out that there are some unbearable mistakes found in the outsource laboratory investigation which may further create problem for practicing physicians. In 2015, there were 53 surveys about the cost awareness of surgical items. They reported that most medical officers and doctors were not much aware about the market price of those items.
There is a need for healthy relationship between the diagnostic centers and the hospital administration which may lead to the revenue generation for the hospital. Hospital administration has to be attentive about the time, staff, equipment, space and other patient care related management [5].

For making healthcare affordable, there should be a collaborative policy research about reducing cost, labor and time for the diagnostic tests. Therefore, Indian medical institutes should promote patient cost audit [6]. Such policy proposals can generate data and give rise to new policies which can enable the state and national health ministries to plan programs and evaluate their impact based on inputs from this policy research proposal.

A systemic study can be planned which could be based on inputs, processes and the output. In the input section, we can analyze number of employees like total number of professors, additional professor, assistant professors, senior residents, junior residents, lab technicians, nurses and class IV and their combined salary. This may include the number of machines or equipment's and their maintenance charges combined together.

A retrospective study can be subsequently carried out by combining 1 year record from radiodiagnostics for the number of tests done, revenue generated, man power and expenses incurred by the department for maintenance from the annual report record sheets. Prospective study can also be carried out as follow-up study. The parameters which are responsible for delay in radiological tests include date of advice for the test and date on which the tests have been conducted. Also, this could help assess delay, pricing, and satisfaction levels of the tests carried out. 


\section{References}

1. Altman, D.J. and R.B. Gunderman, Outsourcing: a primer for radiologists. J Am Coll Radiol, 2008;5(8);893-9.

2. Chasin, B.S., S.P. Elliott, and S.A. Klotz, Medical Errors Arising from Out sourcing Laboratory and Radiology Services. The American Journal of Medicine, 2007;120(9);819.e9-819.e11.

3. Friedman, B., Value based healthcare will drive outsourcing of hospital diagnostic services. Lab Soft News, 2016.

4. Pros and cons of outsourcing laboratory services. Journal of oncology practice, 2006;2(4):162-163.
5. Mrak, R.E., T.G. Parslow, and J.E. Tomaszewski, Outsourcing of Academic Clinical Laboratories: Experiences and Lessons From the Association of Pathology Chairs Laboratory Outsourcing Survey. Academic pathology, 2018;5:2374289518765435-2374289518765435.

6. Anand, A., need for innovation in medical institutions. Annals of neurosciences, 2014;21(1):1-2.

doi: $10.38205 /$ imcr.020234 